\title{
REORIENTAÇÃO DO ENSINO NO SUS: PARA ALÉM DO QUADRILÁTERO, O PRISMA DA EDUCAÇÃO
}

\author{
Carine Vendruscolo ${ }^{1}$ \\ Marta Lenise do Prado ${ }^{2}$ \\ Maria Elisabeth Kleba ${ }^{3}$
}

\section{Resumo}

Propõe-se a reflexão sobre estratégias de reorientação da formação e dos processos de Educação Permanente em Saúde, por meio da observação de instâncias intersetoriais de gestão desses movimentos. Pondera-se sobre a disposição dos sujeitos sociais que integram o conhecido quadrilátero que representa os segmentos integrantes de tais processos (ensino, gestão, atenção e controle social), na dinâmica da formação/educação, representada pela figura. Considera-se que, com essa estrutura, o quadrilátero, por vezes, não se reafirma como tal, ora faltando elementos para encaixar os vértices, ora com a intervenção de outros elementos em um mesmo segmento, configurando-se o que pode-se definir como prisma, para além de quadrilátero. Reflexos são provocados pela ação do quadrilátero, contudo, ressalta-se que as relações dialógicas dos sujeitos geram oportunidades significativas de prismas que traduzem melhor a complexidade, a riqueza e a potência dos movimentos de educação na saúde.

\footnotetext{
1Doutora em Enfermagem pelo Programa de Pós Graduação em Enfermagem da Universidade Federal de Santa Catarina (UFSC). Professora Adjunta do Departamento de Enfermagem da Universidade do Estado de Santa Catarina (UDESC). Rua Barão do Rio Branco 1044 E - Bairro Jardim Itália - Chapecó - SC, Brasil. CEP: 89.801-102. Email: carine.vendruscolo@udesc.br

2Doutora em Filosofia da Enfermagem pelo Programa de Pós-Graduação em Enfermagem da UFSC. Professora do Departamento de Enfermagem e do Programa de Pós Graduação da UFSC. Campos Reitor David Ferreira Lima. Florianópolis/SC, Brasil. CEP: 88840-900. Email: marta.lenise@ ufsc.br

3Doutora em Filosofia pela Universidade de Bremen (Alemanha). Professora do Curso de Enfermagem e dos Mestrados em Ciências da Saúde e em Políticas Sociais e Dinâmicas Regionais da Universidade Comunitária da Região de Chapecó (Unochapecó). Av. Senador Attílio Fontana, 591 E. Bairro Efapi, Chapecó/SC, Brasil. CEP: 89809-000. Email: 1kleba@unochapeco.edu.br
} 
REORIENTAÇÃO DO ENSINO NO SUS: PARA ALÉM DO QUADRILÁTERO, O PRISMA DA EDUCAÇÃO

Palavras-chave: Educação Continuada; Ensino; Serviços de Integração Docente-Assistencial; Sistema Único de Saúde

\section{INTRODUÇÃO}

O Ministério da Saúde (MS) aposta no envolvimento dos trabalhadores da saúde, do controle social, dos gestores e das instituições formadoras com a proposta do Sistema Único de Saúde (SUS), como estratégia eficiente para consolidar a Política de Saúde no Brasil. Partindo do pressuposto de que a educação em saúde precisa emanar de um movimento intersetorial, articulando necessidades de formação e aperfeiçoamento dos trabalhadores, o MS estabelece uma agenda estratégica com o Ministério da Educação (MEC). Por meio da Secretaria de Gestão do Trabalho e da Educação na Saúde (SGTES) lança, em 2004, a Política Nacional de Educação Permanente em Saúde (PNEPS), tendo como uma de suas âncoras a responsabilização dos serviços de saúde com a transformação das práticas dos trabalhadores e na construção de conhecimento comprometido com a realidade social dos sujeitos protagonistas do cuidado (BRASIL, 2004). Entre outras ações, em novembro de 2005, o MS lança o Programa Nacional de Reorientação da Formação Profissional em Saúde (Pró-Saúde), em parceria com o MEC, por intermédio da Secretaria de Educação Superior (SESU) e do Instituto Nacional de Estudos e Pesquisas Educacionais Anísio Teixeira (INEP) (BRASIL, 2007a). Tais iniciativas, integradoras da educação-trabalho, caracterizam-se como ações estruturantes para a reorientação da formação na saúde, in service (lócus da prática) ou pré service (lócus da teoria).

Em 2007, a PNEPS é revisada por meio de uma proposta que resultou na Portaria número 1.996, a qual apresenta novas diretrizes e estratégias para sua implementação. Contudo, em nível de estados e regiões, mantém-se os pressupostos da Portaria número 198/04, como a necessidade de considerar especificidades regionais, superação das desigualdades, necessidades de desenvolvimento para o trabalho em saúde, entre outras (BRASIL, 2004; FERRAZ, 2011). O ideário das $\operatorname{rodas}^{4}$ de Educação Permanente em Saúde (EPS) prevalece como possibilidade de gestão do processo, no qual não há comando vertical e todos os sujeitos (gestores, trabalhadores, estudantes e usuários) influenciam e provocam o

\footnotetext{
4 Relação entre o universo do ensino e o universo do serviço de saúde orienta uma gestão colegiada e, tal qual nas rodas dos jogos infantis, coloca os participantes em uma operação conjunta em que todos usufruem o protagonismo e a produção coletiva (BRASIL, 2004).
} 
movimento de construção do conhecimento (BRASIL, 2005). Para a condução da política, a portaria revisada indicou como estratégia a instalação das Comissões Permanentes de Integração Ensino/Serviço (CIES), definidas como instâncias interinstitucionais permanentes com a função de formulação, condução e desenvolvimento da PNEPS. As CIES, como instâncias intersetoriais e regionais, devem ser compostas pelos gestores da saúde, gestores da educação, trabalhadores do SUS, instituições de ensino com cursos na área da saúde, por meio de seus distintos segmentos, e movimentos sociais ligados à gestão das políticas públicas de saúde e do controle social no SUS (BRASIL, 2007b).

Por sua vez, no âmbito da formação, o Pró-Saúde converge com a proposta de envolvimento recíproco entre instituições de ensino e de serviço, com vistas a planejar e desenvolver atividades articuladas e coerentes com a realidade local, atendendo às diretrizes do SUS e da formação de profissionais orientados para atuar nesse Sistema. Para que ocorra um processo efetivo de integração entre teoria e prática, configurando-se a verdadeira práxis ${ }^{5}$ de que fala Paulo Freire $(2001 ; 2005)$, acredita-se na necessidade de haver participação efetiva de representantes dos segmentos envolvidos no processo, com vistas à construção de propostas conjuntas no âmbito da formação e desenvolvimento de recursos humanos na área da saúde.

As Comissões de Gestão e Acompanhamento Local do Pró-Saúde são instâncias instituídas por meio da Portaria número 3.019/07, com o propósito de acompanhar, monitorar e avaliar os projetos que compõem tal Programa, na esfera do território onde são desenvolvidos. Recomenda-se que as comissões sejam compostas pelo coordenador dos projetos e por representantes do gestor municipal de saúde, do Conselho Municipal de Saúde (CMS), dos profissionais do sistema público de saúde, dos docentes e dos discentes dos cursos envolvidos no Pró-Saúde (BRASIL, 2007c). Espera-se que estas instâncias, com representações dos diferentes sujeitos sociais envolvidos no processo, possam operar como espaços de compartilhamento do poder decisório, mediante movimentos de diálogo e corresponsabilização.

Em estudo reflexivo publicado na Revista Physis, em 2004, Ceccim e Feuerwerker defendem o "quadrilátero da formação para a área da saúde" - ensino, gestão, atenção e controle social -, com vistas à construção e à gestão da educação na saúde no âmbito do SUS.

5 A práxis pode ser compreendida como uma atividade por meio da qual o sujeito modifica a realidade objetiva, de modo reflexivo, remetendo a teoria à prática. Trata-se de um aspecto decisivo para a visão emancipatória da educação, pois está ancorada na interação dialógica e nas mediações que estabelecemos com o outro, com a sociedade e com o mundo (FREIRE, 2001; 2005). 
REORIENTAÇÃO DO ENSINO NO SUS: PARA ALÉM DO QUADRILÁTERO, O PRISMA DA EDUCAÇÃO

O ideário de tal figura pressupõe que cada face estabeleça fluxos e interlocutores específicos, em espaços-tempos e com motivações diferentes, numa trama de conexões.

No intuito de dialogar sobre tais estratégias, com o presente texto, nos atrevemos a provocar algumas reflexões sobre a estratégia de gestão dos processos de formação e EPS, e sobre a maneira como os sujeitos sociais que integram o chamado "quadrilátero" se dispõem, efetivamente, na dinâmica representada por essa figura. As reflexões foram desencadeadas durante o processo de doutoramento de uma das autoras junto ao Programa de Pós Graduação em Enfermagem da Universidade Federal de Santa Catarina (UFSC) (VENDRUSCOLO, 2014) e tiveram continuidade durante a formação da mesma como tutora no Curso EPS em Movimento ${ }^{6}$ do Núcleo de Educação, Avaliação e Produção Pedagógica em Saúde (EducaSaúde), criado na Faculdade de Educação (Faced), da Universidade Federal do Rio Grande do Sul (UFRGS).

\subsection{Refletindo acerca das dimensões do quadrilátero da formação e educação na saúde}

Por meio de ambas as propostas (PNEPS e Pró-Saúde), acredita-se na mudança do modelo assistencial, a qual ressoará na formação e na atenção à saúde somente quando as instâncias corresponsáveis em tais processos estiverem, realmente, comprometidas. A esse efetivo envolvimento dos sujeitos com a construção e a defesa da vida, individual e coletiva, Merhy (2005) chama pedagogia da implicação. Para esse autor, construir processos educativos no cotidiano dos serviços ou da formação, precisa ser uma implacável perseguição de quem deseja, efetivamente, produzir saúde (MERHY, 2005).

A lógica de gestão colegiada prevê igual poder a todas as instituições envolvidas no processo, de tal forma que cada uma delas assuma compromissos com a realidade concreta onde está inserida e, por conseguinte, com a gestão democrática e horizontal do contexto educativo. Nessa direção, a roda, no âmbito da gestão colegiada tem natureza política e crítico-refexiva (CECCIM, FEUERWERKER, 2004). A PNEPS e o Pró-Saúde procuraram manter a coerência teórica de suas ações com tais pressupostos e também com as diretrizes que orientam o SUS e, para tanto, assumiram o referencial denominado de quadrilátero da

6 Esse curso tem como objetivo ativar processos de EPS nos territórios, reconhecendo práticas e saberes existentes no cotidiano do trabalho, incentivando assim, a produção de novos sentidos no fazer saúde, a partir da invenção de práticas de aprender, de cuidar e de viver, destacando a potência do trabalho vivo em ato (EPS EM MOVIMENTO, 2014). 
formação para a área da saúde como estratégia de desenvolvimento da política e também do programa. Com esse propósito, cada um dos vértices desse quadrilátero é composto pela interseção relacional entre os segmentos gestão, atenção, ensino e controle social (CECCIM, FEUERWERKER, 2004; FERRAZ, 2011).

Nossa vivência durante o processo de doutoramento e na formação do Curso EPS em Movimento, assim como outros estudos (KLEBA et al., 2007; TOMBINI, 2010; FERRAZ, 2011), desenvolvidos no âmbito das instâncias colegiadas de gestão dos processos de integração gestão-atenção-ensino-controle social, têm apresentando alguns resultados interessantes, a fim de refletir sobre os papéis e a relação entre os segmentos representados no chamado quadrilátero da formação e educação na saúde. Entre outros, considera-se que as instâncias colegiadas são espaços dialógicos, nos quais são realizados exercícios de cidadania e democracia. Embora existam conflitos, esses não são necessariamente negativos, uma vez que prevalece o diálogo entre os segmentos representados e que este converge para a proposição de ações e para a mudança no processo de gestão da integração ensino-serviçocomunidade.

As experiência junto ao grupo da CIES da Macrorregião Oeste de Santa Catarina têm evidenciado expressiva participação do segmento ensino, tanto por meio de Instituições de Ensino Superior (IES) com curso superior na área da saúde, como dos gestores das Secretarias Municipais de Educação. De outro lado, observa-se pouca representatividade do controle social.

Nessa direção, estudo realizado no oeste de Santa Catarina, com conselhos gestores, aponta que a universidade, para além do compromisso com a comunidade, também apresenta potenciais em prol do empoderamento dos sujeitos, em seus diferentes espaços de interseção. Dessa maneira, participa, efetivamente, na construção de condições favoráveis à vida e à saúde das populações (KLEBA et al., 2007). Assim, a IES precisa se fazer representar junto às diferentes estruturas colegiadas na saúde, quer seja junto ao controle social no SUS, por meio dos CMS, quer seja em instâncias relacionadas aos processos de formação/educação no setor.

No que se refere à implicação da IES, como parte integrante do quadrilátero, em estruturas como a CIES e a instância colegiada de gestão do Pró-Saúde, observam-se atitudes contrastantes entre os sujeitos que a representam. Nossas observações sugerem que, na CIES, o segmento ensino, representado pela universidade, apresenta uma postura, por vezes distante e desarticulada das situações apresentadas por outros segmentos, sobretudo do serviço, mais 
REORIENTAÇÃO DO ENSINO NO SUS: PARA ALÉM DO QUADRILÁTERO, O PRISMA DA EDUCAÇÃO

fortemente representado nessa Comissão. Isso pode ter relação com a falta de efetiva integração entre tais setores (educação/ensino e saúde/serviço) em nível regional, como se observa em outros estudos (TOMBINI, 2010; FERRAZ, 2011), o que dificulta o envolvimento entre ambos, no sentido dialógico e de reconhecimento e compreensão dos diferentes pontos de vista e necessidades. Quanto à estrutura de gestão do Pró-Saúde, são postas situações conflituosas e que envolvem, principalmente, os segmentos ensino e serviço, por conta da defesa de interesses de um e de outro, como por exemplo a alegação do serviço de que os estudantes interferem/atrapalham na rotina do serviço e a reação da universidade, de que o serviço não promove o envolvimento do estudante no cotidiano laboral. Trata-se, afinal, de uma relação de alteridade entre diferentes saberes (teoria e prática), na qual nem sempre flui, naturalmente, o livre movimento dos sujeitos, na direção da práxis. O que se percebe são interesses individuais, que por vezes, se sobrepõem à prerrogativa constitucional ou ao ideário que atribui ao SUS a formação de recursos humanos na área da saúde (BRASIL, 1988). Tais interesses estão implicados nessa construção, e emanam dos diferentes sujeitos que compõem a IES - docentes, discentes, gestores, áreas da saúde, núcleos do saber.

Nesse contexto, sobre alteridade, vale destacar Larrosa (2011, p. 05), que conceitua tal expressão como um acontecimento ou, dito de outro modo, o passar de algo que não sou eu, por meio da experiência.

E “algo que não sou eu” significa também algo que não depende de mim, que não é uma projeção de mim mesmo, que não é resultado de minhas palavras, nem de minhas ideias, nem de minhas representações, nem de meus sentimentos, nem de meus projetos, nem de minhas intenções, que não depende nem do meu saber, nem de meu poder, nem de minha vontade. "Que não sou eu" significa que é "outra coisa que eu", outra coisa do que aquilo que eu digo, do que aquilo que eu sei, do que aquilo que eu sinto, do que aquilo que eu penso, do que eu antecipo, do que eu posso, do que eu quero.

A participação dos usuários em instâncias de decisão na área da saúde é um direito garantido por lei no Brasil, mediante ação denominada de controle social, exercida em especial nos conselhos gestores (BRASIL, 1990). O usuário aqui é o cidadão que faz uso do serviço (e nesse sentido todos o fazem), mas que, para dar sentido à representação, não deveria pertencer a um dos outros lados do quadrilátero (gestão, atenção ou ensino). Em tese, qualquer representante de qualquer um dos vértices do quadrilátero poderia ser duplo representante, o que, todavia, para garantir e respeitar o princípio doutrinário da lei, deveria ser evitado. O que se percebe, portanto, nas instâncias de gestão dos processos de educação em saúde, é que a participação do controle social é pouco frequente e que, quando ocorre, 
nem sempre o representante é o usuário (no sentido desejado) do serviço, mas outro membro do CMS (muito frequentemente, profissionais de saúde ou representante da gestão).

Vale lembrar que o CMS, por conta da paridade, é composto por usuários, prestadores de serviço, trabalhadores ou gestores. Nesse sentido, a participação do controle social no quadrilátero apresenta uma distorção - um desvio de prisma - o que acaba por comprometer a paridade que se deseja nesse processo.

A implicação do segmento gestor com os processos de educação/formação é estimulada por meio de mecanismos tais como a instituição do poder deliberativo dos Colegiados de Gestão Regional (CGR) ${ }^{7}$, propondo o envolvimento dos secretários municipais de saúde com as questões referentes à EPS nos territórios de saúde pelos quais são responsáveis. Assim, todas as ações propostas ou desenvolvidas pelas CIES necessitam da aprovação dos colegiados (BRASIL, 2007b). Nas CIES ou outras estruturas de gestão colegiada relativas ao ensino, como as Comissões de Gestão e Acompanhamento Local do Pró-Saúde, os gestores (Secretários Municipais de Saúde) são representados, via de regra por coordenadores de serviços no âmbito da Secretaria. Isso também ocorre em relação à representação da gestão estadual, no caso das CIES. Não se percebe, entretanto, da parte de muitos dos gestores um maior reconhecimento e valorização do papel que seus representantes exercem nesses espaços, garantindo o apoio necessário e os encaminhamentos implicados a partir das decisões tomadas nesses fóruns.

Finalmente, quanto aos trabalhadores e seu desenvolvimento na perspectiva do SUS, é conhecida a insuficiência de preparo para o desempenho das atividades no Sistema, apesar dos avanços registrados durante o período histórico de sua vigência. É consenso, entre os críticos da saúde e da educação, a hegemonia do modelo de atenção centrado na racionalidade biomédica, o qual reflete no ensino na área da saúde, assim como as práticas pedagógicas, fundamentadas em metodologias pouco problematizadoras ou críticas, e que também interferem no cuidado em saúde (HADDAD et al., 2008). Soma-se a isso, as dificuldades de integração ensino-serviço, antes problematizadas.

Ao criar-se estratégias de estruturação de processos educativos que assegurem novas práticas sanitárias, superando os modelos tradicionais centrados nas abordagens de doença e riscos, é preciso considerar a adequação do perfil profissional e o desenvolvimento de iniciativas de valorização dos trabalhadores. Nessa perspectiva, a PNEPS e também o PróSaúde propõem a aprendizagem significativa, a partir da reflexão sobre as práticas, 
REORIENTAÇÃO DO ENSINO NO SUS: PARA ALÉM DO QUADRILÁTERO, O PRISMA DA EDUCAÇÃO

intensificada na interação entre sujeitos do ensino e do serviço, possibilitando olhares e saberes diversos, com vistas à sua reorientação. O aprender e o ensinar, incorporados ao cotidiano das organizações e do processo laboral e aos problemas enfrentados na realidade, somados aos conhecimentos e experiências prévias dos sujeitos, prometem garantir a possibilidade de transformar as práticas (FREIRE, 2001; 2005; BRASIL, 2007b).

Integrar pressupõe, de certo modo, estar aberto ao diálogo e considerar as divergências, com vistas à reconstrução das práticas. Tombini (2010) identifica diferentes níveis de integração ensino-serviço, insinuados no envolvimento/papel dos trabalhadores, podendo se restringir à aceitação (ou não) do convívio com a IES (docentes/discentes). Nesse sentido, o profissional da saúde muitas vezes apresenta um nível de aceitação "passiva”, comum quando este não se envolve nas construções da IES, permanecendo como mero expectador do processo. De outro lado, o profissional pode estar realmente envolvido no processo e até comprometido com ele, de acordo com o nível de responsabilização que manifesta, não só a partir do "seu querer", mas em relação à "gerência do querer" dos demais integrantes da equipe de saúde e da própria comunidade.

Contextualizada a importância de cada um dos segmentos do quadrilátero em relação a sua participação e representação no processo de formação/educação, convém provocar algumas reflexões sobre a integração entre os lados que o compõem. Com isso, retomamos o pensar sobre o significado da ação de representar. Podemos dizer que trata-se de uma concepção segundo a qual os indivíduos, nomeados para agir no interesse de grupos, necessitam tempo para se apropriar do processo, além de habilidades de comunicação para se relacionar com os demais e para negociar (ABERS; KECK, 2008). Ao discutir a dinâmica de algumas instâncias colegiadas em relação aos laços recíprocos, supostamente mantidos entre os membros representantes e os grupos representados, Abers e Keck (2008) demonstram que a legitimidade da representação depende de uma relação de delegação e retorno entre ambos, na qual o representante deve ser capaz de reconstruir o raciocínio dos representados, defendendo-o de maneira eficaz. Convém destacar também, os conflitos que podem ser desencadeados no caso de uma representação "mal feita", na qual não houve consenso entre representantes e representados. As ligações, por vezes frágeis, entre os sujeitos representantes e representados, ou mesmo os problemas relacionados à importância (poder) do representado junto à sua entidade de origem - e em nome da qual supostamente fala - implica na tomada de decisões que podem comprometê-la. Com tais argumentos, esses autores propõem um maior compromisso com a participação em arenas deliberativas, como os conselhos gestores, 
a fim de transformar a prática e contribuir com a democracia. Sinalizam, todavia, que é preciso atentar para não deixar escapar a dimensão da criatividade, congelando as relações que se estabelecem, na defesa de homogeneidade de posições, sem dar vasão ao livre movimento dos indivíduos, mediante seus diferentes pontos de vista. Esta atitude libertária pode dar vasão a "relações fecundas", originárias de elementos que não são fruto do pensamento de um ou de outro sujeito, mas sim, inovadoras.

Todos os sujeitos que, de alguma maneira, estão implicados com a produção da saúde, representam certos espaços, e governam o cotidiano do trabalho em saúde, dado o grau de liberdade que existe no seu agir (FRANCO; MERHY, 2012). Feita a reflexão sobre as relações, fecundas ou não, entre eles, vamos aos fatos. Primeiro, estamos convencidos que a representação dos segmentos nas instâncias de gestão colegiada dos processos educativos, como é o caso da CIES e das Comissões de Gestão e Acompanhamento Local do Pró-Saúde, ocorre de maneira heterogênea. A gestão da saúde e controle social são os segmentos que menos se envolvem, ao passo que ensino e serviço, entre acordos e divergências, têm construído um caminhar coletivo, representados, no âmbito do seu segmento, da seguinte maneira: no ensino estão representados gestores da IES, docentes e estudantes; e ainda, no caso das CIES, Secretários Municipais de Educação; no serviço, fazem-se presentes gestores, profissionais/trabalhadores. O segmento gestão - no caso, ligado à saúde - embora menos presente, quando representado, se faz por meio de coordenadores de departamentos (atenção básica, vigilância epidemiológica, entre outros), mediante indicação do Secretário Municipal de Saúde, e nunca mediante a sua presença, em pessoa. Já o controle social, como problematizamos, tem no usuário/cidadão, um sujeito poucas vezes representado, de fato. A figura a seguir (Figura 1), procura ilustrar esses aspectos. 


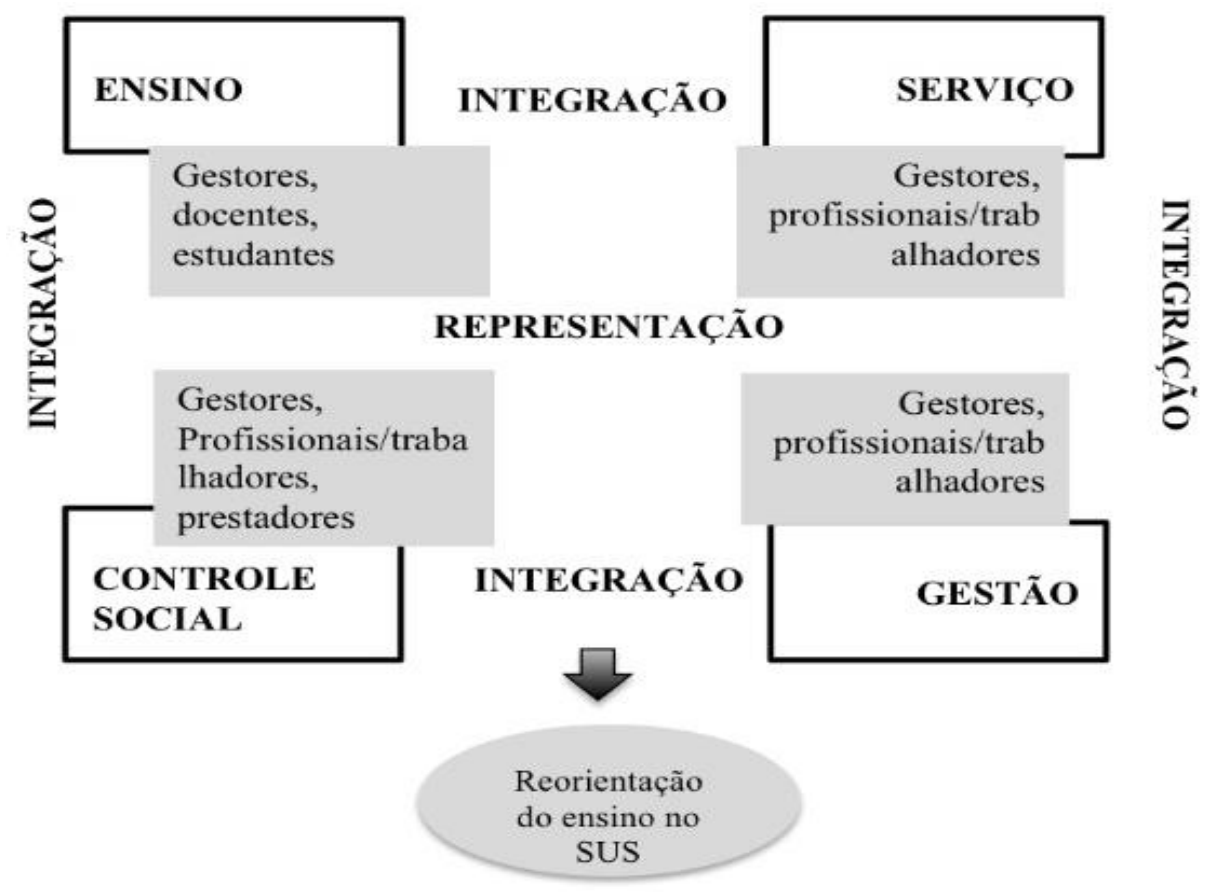

Figura 1: Diagrama ilustrativo da reorientação do ensino no SUS.

Dessa maneira, cada um dos sujeitos e segmentos representados - educadores, usuários, gestores e trabalhadores - estão implicados em realidades e situações diversas, as quais repercutem na maneira de exercer essa representação. Nas IES, temos o maior controle do processo de formação, sendo que os sujeitos que exercem a representação com mais afinco são docentes, havendo pouca participação de estudantes e gestores desse segmento. $\mathrm{O}$ trabalhador, representado nos segmentos gestão, serviço e, via de regra, controle social, tem o domínio do cenário das práticas e enfrenta problemas advindos do processo de trabalho, alinhados a uma formação biologicista e a uma comunidade com necessidades básicas de atenção à saúde, além do estranhamento diante da presença da IES (docentes/discentes), compreendendo-os muitas vezes, como intrusos.

Com essa configuração, esse quadrilátero, por vezes, não se reafirma como tal, geometricamente falando, pois, ora faltam elementos para encaixar os vértices, ora percebe-se a intervenção de outros elementos em um mesmo segmento, configurando-se o que poderíamos definir como um prisma, para além de um quadrilátero.

O que se espera, todavia, é que profissionais, estudantes, docentes, pesquisadores, gestores do ensino e do serviço, e comunidade, ao formularem pactos e políticas visando à reorientação do ensino na saúde, sejam capazes de constituírem-se como redes sociais, comprometendo-se com o desenvolvimento do território e com a produção do trabalho, bem como com a promoção da saúde (REIBNITZ et. al., 2009). 
Acredita-se que a interação efetiva entre os segmentos que fazem parte do complexo sólido que tentamos representar, produz nos sujeitos maior compromisso, por meio de relações de vínculo e responsabilização entre estudantes, usuários, professores, profissionais, gestores e outros parceiros na produção dos processos de educação e de cuidado em saúde. O prisma pode ser utilizado para refletir raios luminosos e, utilizando-se dessa metáfora, podemos supor que ainda há muitas questões para serem ajustadas em relação a esses espaços de aprendizado da democracia. Contudo, se já são perceptíveis os reflexos provocados pela ação do quadrilátero, certamente as rodas tem gerado oportunidades significativas para a conformação de, por que não dizer, prismas da formação e da educação em saúde, que traduzem a complexidade, a riqueza e a potência dos processos de educação permanente na saúde.

\title{
2 CONSIDERAÇÕES FINAIS
}

O exercício de democracia, de inclusão e de responsabilização dos sujeitos que estão implicados na construção do SUS é o grande desafio do Pró-Saúde, da PNEPS e de outras ações voltadas à reorientação e qualificação dos processos de formação na saúde. Os sujeitos que compõem a estrutura das instâncias de gestão e coordenação de tais ações - seja percebida como quadrilátero, seja como prisma - se reinventam gradativamente e, nesta perspectiva, também reinventam a maneira de produzir saúde.

$\mathrm{Na}$ área do ensino em saúde, o que se percebe é que os sujeitos estão cada vez mais convictos de que a mudança é possível, desde que a integração dos elementos implicados se consolide como parceria no seu sentido mais amplo, sejam os segmentos representados por estudantes, docentes, profissionais, gestores, usuários ou outros sujeitos, desde que o foco seja a qualidade da atenção à saúde coletiva.

\section{EDUCATION IN REORIENTATION SUS: BEYOND THE KNOWN FOUR-WAY APPROACH, THE EDUCATION PRISMA}

\author{
Abstract


REORIENTAÇÃO DO ENSINO NO SUS: PARA ALÉM DO QUADRILÁTERO, O PRISMA DA EDUCAÇÃO

It is proposed to reflect on the strategies for reorienting the training and processes of Continuing Education in Health, by observing of intersectoral management instances of these movements. Ponders about the move of social subjects that comprise the known four-way approach (teaching, management, care and social control) in the formation, the dynamics of training/education, represented by the figure. It is considered that with this structure, the fourway approach, sometimes not reaffirmed as such, now missing elements to fit the vertices, sometimes with the use of other elements in the same segment, setting up what can be defined as "prism", in addition to four-way approach. Reflections are caused by the action of the fourway approach, but, in addition, the dialogical relationship of subjects generate significant opportunities of the prisms that translate best complexity, the wealth and the power of education movements in health.

Keywords: Continuing Education; Teaching; Teaching Care Integration Service; Unified Health System

\section{EDUCACIÓN EN SUS REORIENTACIÓN: MÁS ALLÁ DEL CUADRILÁTERO, EDUCACIÓN PRISMA}

\section{Resumen}

Se propone reflexionar sobre las estrategías para la reorientación de la formación y los procesos de Educación Continua en Salud, mediante la observación de las instancias de gestión intersectorial de estos movimientos. Ponder está en la disposición de los sujetos sociales que componen el conocido cuadrilátero que representa los segmentos de los miembros de tales procesos (docencia, gestión, atención y control social), la dinámica de la formación/ducación, representados por la figura. Se considera que con esta estructura, el cuadrilátero, a veces no es caracterizado como tal, que le faltan elementos para adaptarse a los vértices, a veces con el uso de otros elementos en el mismo segmento, formando lo que puede ser definido como "prisma", más allá de uno cuadrilátero. Reflexiones son causadas por la acción del cuadrilátero, pero, además, la relación dialógica de los sujetos puede generar oportunidades significativas de prismas que mejor traducen la complejidad, la riqueza y el poder de los movimientos de educación en salud. 
Palabras-clave: Educación Continua; Enseñanza; Servicios de Integración Docente Asistencial; Sistema Único de Salud

\section{REFERÊNCIAS}

ABERS, R. N.; KECK, M. E. Representando a Diversidade: estado, sociedade e "relações fecundas" nos conselhos gestores. Caderno $C R H$, Salvador: Universidade Federal da Bahia, vol. 21, n. 52, p. 99-112, jan/abr. 2008.

BRASIL. Congresso Nacional. Constituição da República Federativa do Brasil. Promulgada em 05 de outubro de 1988. [documento internet] 1988. Disponível em: < http://www.planalto.gov.br/legislacao/>. Acesso em: 12 Set. 2012.

. Ministério da Saúde. Lei $n^{o} 8.142$, de 28 de dezembro de 1990. Dispõe sobre a participação da comunidade na gestão do Sistema Único de Saúde (SUS) e sobre as transferências intergovernamentais de recursos financeiros na área da saúde e dá outras providências. [documento internet] 1990. Disponível em: <http://portal.saude.gov.br>. Acesso em: 12 Set. 2012.

Ministério da Saúde. Ministério da Educação. Portaria GM/MS no 198/04, de 13 de fevereiro de 2004. Institui a Política Nacional de Educação Permanente em Saúde - como estratégia do Sistema Único de Saúde para a formação e o desenvolvimento de trabalhadores para o setor e dá outras providências. [documento internet] 2004. Disponível em: <http://www.saude.gov.br/sgtes>. Acesso em: 12 Jul. 2012.

Ministério da Saúde. Ministério da Educação. A educação permanente entra na roda: pólos de educação permanente em saúde: conceitos e caminhos a percorrer. Brasília, 2005.

Ministério da Saúde. Ministério da Educação. Programa Nacional de Reorientação da Formação Profissional em Saúde - Pró-Saúde: objetivos, implementação e desenvolvimento potencial. Brasília: Ministério da Saúde; 2007 a. 86 p. (Série C. Projetos, Programas e Relatórios).

Ministério da Saúde. Ministério da Educação. Portaria GM/MS n $n^{o}$ 1.996/07, de 20 de agosto de 2007. Dispõe sobre as diretrizes para a implementação da Política Nacional de Educação Permanente em Saúde. [documento internet] 2007b. Disponível em: <http://www.saude.gov.br/sgtes $>$. Acesso em: 24 Ago. 2007.

Ministério da Saúde. Ministério da Educação. Portaria Interministerial no. 3.019, de 26 de novembro de 2007. Dispõe sobre o Programa Nacional de Reorientação da Formação Profissional em Saúde - Pró-Saúde para os cursos de graduação da área da saúde. Brasília, 2007c. 
REORIENTAÇÃO DO ENSINO NO SUS: PARA ALÉM DO QUADRILÁTERO, O PRISMA DA EDUCAÇÃO

Ministério da Saúde (BR). Secretaria de Gestão Estratégica e Participativa. Decreto no 7.508, de 28 de junho de 2011: regulamentação da Lei no 8.080/90. Brasília (DF): MS; 2011. 16 p. (Série E. Legislação de Saúde).

CECCIM, R. B.; FEUERWERKER, L. M. C. O Quadrilátero da Formação para a Área da Saúde: Ensino, Gestão, Atenção e Controle Social. PHYSIS: Revista Saúde Coletiva, Rio de Janeiro: UFJF, vol. 14, n. 1, p. 41-65, 2004.

EPS EM MOVIMENTO. Entrada, Apresentação. 2014. Disponível em: <http://eps.otics.org/material/entrada-apresentacao/entrada-apresentacao/>. Acesso em: 05 Set. 2015.

FERRAZ, Fabiane. Contexto e processo de desenvolvimento das Comissões Permanentes de Integração Ensino Serviço: perspectivas dos sujeitos sociais pautada na concepção dialógica de Freire. 2011. 421 f. Tese (Doutorado em Enfermagem) - Universidade Federal de Santa Catarina, Florianópolis.

FRANCO, T. B.; MERHY, E. E. Cartografias do trabalho e cuidado em saúde. Tempus: Actas de Saúde Coletiva, Brasília: UNB, vol. 6, p. 151-163, 2012.

FREIRE, Paulo. Conscientização: teoria e prática da libertação: uma introdução ao pensamento de Paulo Freire. São Paulo: Centauro, 2001, 116p.

. Pedagogia do oprimido. 41. ed. Rio de Janeiro: Paz e Terra, 2005.

HADDAD, A. E. et AL. Política Nacional de Educação na Saúde. Revista Baiana de Saúde Pública, Salvador, vol. 32, n. 1, p. 98-114, 2008.

KLEBA, M. E.; COMERLATTO, D.; COLLISELli, L. Promoção do empoderamento com conselhos gestores de um Pólo de Educação Permanente em Saúde. Texto \& Contexto em Enfermagem, Florianópolis: Universidade Federal de Santa Catarina, vol. 16, n.2, p. 335-42, 2007.

LARROSA, Jorge. Experiência e Alteridade em Educação. Revista Reflexão e Ação, Santa Cruz do Sul: UNISC, vol. 19, n. 2, p. 4-27, jul./dez. 2011.

MERHY, Emerson Elias. O desafio que a educação permanente tem em si: a pedagogia da implicação. Interface - Comunicação, Saúde e Educação, Botucatu: Unesp, vol. 9, n. 16, p.161-77, set.2004/fev.2005.

REIBNITZ, K. S. et al. Educação permanente em saúde: contribuição para a consolidação do Sistema Único de Saúde In: LEITE, M. M. J.; MARTINI, J. G.; FELLI, V. E. A. (Orgs.). PROENF - Programa de Atualização em Enfermagem - Saúde do Adulto. 1. ed. Porto Alegre - RS: Artmed/Panamericana Editora, 2009, vol. 4.

TOMBINI, Larissa Hermes Thomas. Educação permanente e integração ensino-serviço na perspectiva dos enfermeiros do serviço. 2010. 177p. Dissertação (Mestrado em Enfermagem) - Universidade Federal de Santa Catarina, Florianópolis. 
VENDRUSCOLO, Carine. Integração ensino-serviço: movimentos das instâncias de gestão nos processos de reorientação da formação profissional na saúde. 2014. 352p. Tese

(Doutorado em Enfermagem) - Universidade Federal de Santa Catarina, Florianópolis.

Data de recebimento: 27/11/2014

Data de aceite: 03/05/2016 